\title{
PRODUCCIÓN Y CARACTERIZACIÓN QUÍMICA DE BIOCHAR A PARTIR DE RESIDUOS ORGÁNICOS AVÍCOLAS
}

\author{
Enmer Trujillo A..$^{*}$, Christian Eduardo Valencia A.a, María Cecilia Alegría A.a, \\ Alejandrina Honorata Sotelo M. ${ }^{\mathrm{b}}$, Mary Flor Césare C. ${ }^{\mathrm{a}}$
}

\begin{abstract}
RESUMEN
Los sistemas avícolas intensivos generan residuos orgánicos (gallinaza y pollinaza), las cuales, al ser utilizadas frescas causan impactos negativos al ambiente, sin embargo, estos residuos orgánicos son ricos en nutrientes que pueden ser utilizados como enmiendas del suelo, siendo la producción de biochar una alternativa para su aprovechamiento. En esta investigación, la producción del biochar se efectuó con un horno pirolizador $(5 \mathrm{~kg}$ de capacidad). Midiéndose en la etapa de producción los parámetros: temperatura, rendimientos y densidad del material resultante. Asimismo, se caracterizó tanto la materia prima (gallinaza y pollinaza) como el biochar obtenido, determinándose: $\mathrm{pH}$, humedad, cenizas, carbono orgánico total y contenidos de los macro y micronutrientes. Al evaluar las propiedades del biochar se obtuvieron diferencias estadísticamente significativas entre la pollinaza y gallinaza en rendimientos, densidad, $\mathrm{pH}$, humedad, cenizas, nitrógeno, fósforo total, calcio, magnesio y fierro. El biochar fue sometido a pruebas de bioensayo para determinar el índice de crecimiento de las semillas y el crecimiento de los tallos de los primeros diez días. El índice de germinación del biochar de la gallinaza resultó significativo con el tratamiento del $3 \%$ y como consecuencia la longitud de crecimiento de los tallos de las plántulas fue significativa.
\end{abstract}

Palabras clave: biochar, pirólisis, caracterización, residuos orgánicos avícolas.

\section{PRODUCTION AND CHEMICAL CHARACTERIZATION OF BIOCHAR FROM ORGANIC AGRICULTURAL WASTE}

\begin{abstract}
\footnotetext{
${ }^{a}$ Departamento Académico de Química. Facultad de Ciencias. UNALM Lima - Perú.

*e-mail: perugreen@hotmail.it

b Departamento Académico de Nutrición. Facultad de Zootecnia UNALM Lima - Perú.
}

The intensive poultry systems generate organic waste (poultry manure and poultry litter); which, when used fresh, cause negative impacts to the environment, however, these organic residues are rich in nutrients that can be used as soil amendments, being the biochar production an alternative for their use. In this investigation, the biochar production was carried out with 
490 Enmer Trujillo A., Christian Eduardo Valencia A., María Cecilia Alegría A., Alejandrina Honorata Sotelo M...

a pyrolyzing furnace ( $5 \mathrm{~kg}$ capacity). Measuring in the production stage the parameters: temperature, yields and density of the resulting material. Likewise, the raw material (chicken and poultry litter) and the biochar obtained were characterized, determining: $\mathrm{pH}$, humidity, ash, total organic carbon and contents of the macro and micronutrients. When evaluating the properties of the biochar, statistically significant differences were obtained between the poultry litter and chicken manure in yields, density, $\mathrm{pH}$, humidity, ash, nitrogen, total phosphorus, calcium, magnesium and iron. The biochar was subjected to bioassay tests to determine the growth rate of the seeds and the growth of the stems of the first ten days. The germination rate of the biochar of the chicken manure was significant with the treatment of $3 \%$ and as a consequence the length of growth of the stems of the seedlings was significant.

Key words: biochar, pyrolysis, characterization, poultry organic waste.

\section{INTRODUCCIÓN}

El deterioro progresivo del medio ambiente es uno de los temas de mayor preocupación. El desarrollo y crecimiento económico y tecnológico de la sociedad han conllevado a un incremento exponencial de los residuos generados ${ }^{1}$, así la Ley de Gestión Integral de Residuos Sólidos (D.L 1278), indica que todos los residuos provenientes de procesos agro industriales sean valorizados al ser transformados en compost ${ }^{2}$, biochar, violes u otros productos. Así, autores $^{3}$ definen al biochar o biocarbón como el producto obtenido de la descomposición térmica de materiales orgánicos con escaso o limitado suministro de oxígeno (pirólisis), a temperaturas inferiores a $\operatorname{los} 700^{\circ} \mathrm{C}$, siendo este usado para fines agrícolas como mejorador del suelo, (retener agua y/o almacenar carbono) lo cual lo diferencia del carbón (combustible) y del carbón activado.

Dado que la industria avícola es una de las actividades que genera gran cantidad de residuos al final del periodo (Gallinaza: entre $20-28 \mathrm{~kg}$ /ave y la pollinaza: entre 1,5 y $2 \mathrm{~kg}$ por ave) ${ }^{4}$, cuya gestión inadecuada, podría contaminar el agua y el aire como fuente de proliferación y diseminación de plagas, enfermedades de importancia sanitaria humana y animal; además, contribuye el cambio climático y afecta los ecosistemas. Por ello, una gestión adecuada de estos residuos contribuye el ahorro de materias primas, la conservación de los recursos naturales y el desarrollo sostenible ${ }^{5}$.

Así, la producción de biochar de estos residuos permitirá cumplir la normativa vigente, al valorizarse como enmienda orgánica, lo que permitiría su posible uso en la restauración y recuperación de los suelos degradados, o simplemente la aplicación puntual en un suelo con unas características definidas y bajo unos objetivos de enmienda establecidos ${ }^{1}$. El empleo de biocarbón puede ser una posibilidad para contribuir a paliar la pérdida de fertilidad y la declinación de las propiedades químicas, físicas y biológicas de los suelos y particularmente la disminución de la materia orgánica; también como material de encalado ya que tienen los $\mathrm{pH}$ altos ${ }^{3}$. 
Las propiedades del biochar están influenciadas por las condiciones de pirólisis como: la temperatura y tiempo de tratamiento, velocidad de calentamiento y características del horno ${ }^{6}$. También están influenciadas por las características de la materia prima como son el carbono total, el carbono fijo y los elementos minerales del biochar, mientras el área superficial del biochar y el pH están influenciados por los tratamientos a mayores o menores temperaturas ${ }^{7}$.

Por ello, la presente investigación tuvo como objetivos: producir biochar con residuos avícolas mediante la pirólisis lenta, caracterizar químicamente tanto la materia prima como el biochar obtenido, finalmente realizar ensayos toxicológicos para determinar la dosis adecuada de biochar a aplicar al suelo, mediante el test de crecimiento del Raphanus sativus L.

\section{PARTE EXPERIMENTAL}

\section{Materiales y métodos}

Los residuos avícolas (gallinaza y pollinaza) utilizados en la producción del biochar provienen de las granjas avícolas de la empresa San Fernando S.A. ubicados en el distrito de Huaral y Lurín. Se seleccionó granjas representativas de producción de gallinas ponedoras y de los pollos de engorde de la empresa en la zona costera del Perú. El horno pirolítico (equipo usado para la producción del biochar) fue diseñado y fabricado en base a la necesidad de producción del biochar necesarias para las muestras requeridas para la investigación.

Su diseño y su elaboración se muestran en la figura 1, se encuentra compuesto por dos componentes. Un tambor externo en forma rectangular que tiene en común dos cámaras para la producción de biochar con perforaciones para la liberación de energía o gases volátiles de combustión de la materia prima de entrada. El otro cilindro interno con finalidad de alimentar biomasa residual para el incremento de temperatura de pirólisis condicionados con perforaciones regulables de aireación y tamizado para la recolección de cenizas. El primer tambor externo tiene medidas de 0,50 x 0,30 x 0,60 cm. El cilindro interno tiene medida de 0,60 de altura por un radio de $0,25 \mathrm{~cm}$.

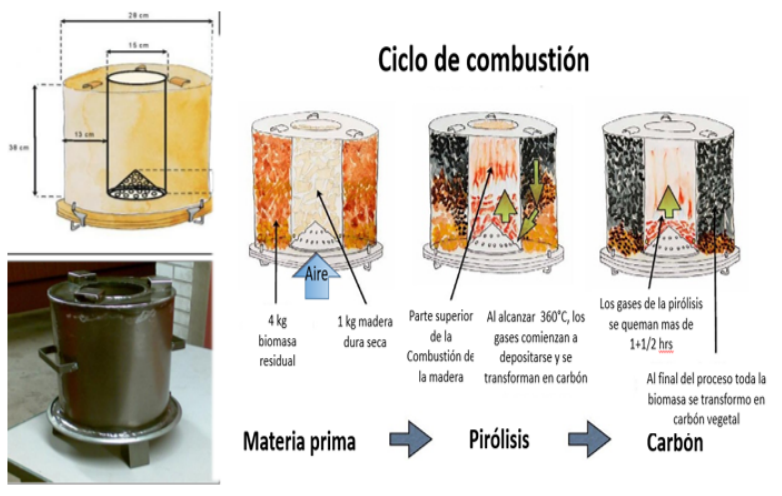

Figura 1. Horno pirolítico usado en la producción del biochar.

Fuente: Ciclo de combustión de estufa pirolítico a nivel familiar ${ }^{8}$. 
492 Enmer Trujillo A., Christian Eduardo Valencia A., María Cecilia Alegría A., Alejandrina Honorata Sotelo M...

En las pruebas de bioensayo de toxicidad se usó semillas de rábano de las especies Raphanus sativus L., se realizó en base a los principios de prueba estática de toxicidad aguda ${ }^{9,10}$ en las que se pueden evaluar los efectos fitotóxicos del biochar de 4 concentraciones $(1 \%, 3 \%$, $5 \%$, y $7 \%$ ) en el proceso de germinación de las semillas y en el desarrollo de las plántulas durante los diez primeros días de crecimiento ${ }^{11}$.

Producción del biochar. El proceso productivo se realizó de la siguiente manera:

- Se controlaron las entradas de aire en ambos tambores o cámaras, debido a que la pirólisis se lleva a cabo en condiciones de ausencia de oxígeno, siendo el control principal del suministro del oxígeno sobre la cámara interna para la combustión térmica.

- Se llenó de biomasa residual a convertir en biochar. La cantidad dependió del tipo y cantidad necesaria de material sin compactar en las cámaras.

- La cámara externa se llenó con material combustible de podaduras (leña, ramas secas, hojas) Una vez completado todo el espacio libre se suministró el fuego para completar la combustión en la generación del biochar.

- Durante el proceso de pirólisis se monitorizó constantemente la temperatura con un termómetro infrarrojo tanto en el tope, medio y en el fondo de la unidad exterior. El proceso tiene una duración promedio de tres horas. Luego de su enfriamiento se retira para el pesado y separación de muestras para el análisis de laboratorio.

\section{Caracterización de los residuos y del biochar}

Se caracterizó la materia prima a utilizar y el biochar resultante. La tabla 1 muestra los resultados del contenido de análisis y la metodología aplicada de las características morfológicas y estructurales de ambos materiales.

Tabla 1. Relación de contenido de análisis y metodología aplicada.

\begin{tabular}{|c|c|c|c|c|}
\hline $\mathbf{N}^{\circ}$ & $\begin{array}{c}\text { Contenido - } \\
\text { Nutrientes } \\
\end{array}$ & Unidad & Metodología & \\
\hline 1 & $\mathrm{pH}$ & & AOAC 973,04 Cap. 2 Ed 19 Pág. 19. 53-54 & 2012 \\
\hline 2 & Humedad & $(\mathrm{g} / 100 \mathrm{~g})$ & NTP 311,012 1970 & \\
\hline 3 & Cenizas & $(\mathrm{g} / 100 \mathrm{~g})$ & AOAC 967,04 Cap. 2 Ed 19 Pág. 19. 54 & 2012 \\
\hline 4 & Carbono total $(\mathrm{C})$ & $(\mathrm{g} / 100 \mathrm{~g})$ & El analizador de COT SHIMADZU L5000 & \\
\hline 5 & Nitrógeno total $(\mathrm{N})$ & $(\mathrm{g} / 100 \mathrm{~g})$ & AOAC 955,04 Cap. 2 Ed 19 Pág. 19. 13-14 & 2012 \\
\hline 6 & Fósforo total (P) & $(\mathrm{mg} / \mathrm{kg})$ & AOAC 958,01 Cap. 2 Ed 19 Pág. 19. 5-6 & 2012 \\
\hline 7 & Potasio $(\mathrm{K})$ & $(\mathrm{mg} / \mathrm{kg})$ & AOAC 983,02 Cap. 2 Ed 19 Pág. 19. 24-27 & 2012 \\
\hline 8 & Sodio $(\mathrm{Na})$ & $(\mathrm{mg} / \mathrm{kg})$ & AOAC 965,09 Cap. 2 Ed 19 Pág. 19. 29-31 & 2012 \\
\hline 9 & Calcio $(\mathrm{Ca})$ & $(\mathrm{mg} / \mathrm{kg})$ & AOAC 965,09 Cap. 2 Ed 19 Pág. 29. 29-31 & 2012 \\
\hline 10 & Magnesio (Mg) & $(\mathrm{mg} / \mathrm{kg})$ & AOAC 965,09 Cap. 2 Ed 19 Pág. 29. 29-31 & 2012 \\
\hline 11 & Cobre $(\mathrm{Cu})$ & $(\mathrm{mg} / \mathrm{kg})$ & AOAC 965,09 Cap. 2 Ed 19 Pág. 29. 29-31 & 2012 \\
\hline 12 & Fierro $(\mathrm{Fe})$ & $(\mathrm{mg} / \mathrm{kg})$ & AOAC 965,09 Cap. 2 Ed 19 Pág. 29. 29-31 & 2012 \\
\hline 13 & Manganeso (Mn) & $(\mathrm{mg} / \mathrm{kg})$ & AOAC 965,09 Cap. 2 Ed 19 Pág. 29. 29-31 & 2012 \\
\hline 14 & Zinc (Zn) & $(\mathrm{mg} / \mathrm{kg})$ & AOAC 965,09 Cap. 2 Ed 19 Pág. 29. 29-31 & 2012 \\
\hline
\end{tabular}


Se determinó la densidad aparente y el porcentaje de rendimiento del biochar resultante mediante:

$$
\begin{gathered}
\text { Densidad aparente }=\frac{\text { Masa de materia seca }}{\text { Volumen del biochar resultante }} \\
\% \text { Rendimiento }= \\
\text { Masa del biochar } \quad \text { x } 100
\end{gathered}
$$

Prueba de bioensayo. Se adaptado el método10 para la prueba del biochar ${ }^{11}$ : Evaluándose el porcentaje de germinación y longitud del crecimiento del tallo, se realizaron los cálculos matemáticos con la finalidad de obtener el índice de germinación, para ello se calcularon dos índices: el porcentaje de germinación relativo (PGR) y crecimiento de tallo relativo (CTR) de cada tratamiento.

$$
\begin{gathered}
\text { PGR }=\frac{\mathrm{N}^{\circ} \text { de semillas germinadas en maceta }}{\mathrm{N}^{\circ} \text { de semillas sembradas en maceta }} \times 100 \\
\mathrm{CTR}=\frac{\text { Elongación del tallo de la plántula por maceta }}{\mathrm{N}^{\circ} \text { de semillas sembradas en maceta }} \times 100 \\
\mathrm{IG}=\frac{\mathrm{PGR} \times \mathrm{CTR}}{100}
\end{gathered}
$$

Dónde:

PGR: Porcentaje de germinación relativo.

CTR: Crecimiento de tallo relativo.

IG: Índice de germinación

Los autores ${ }^{10,11}$ recomiendan, para valores de $\mathrm{IG} \geq 80 \%$, el extracto del analito tratado no presenta sustancias fitotóxicas o están en muy baja concentración; así, valores de $\mathrm{IG} \leq 50 \%$, esto no indicaría que hay una fuerte presencia de sustancias fitotóxicas y sí se obtiene como resultados valores entre $50 \%$ y $80 \%$ se interpretaría como la presencia moderada de estas sustancias.

\section{RESULTADOS Y DISCUSIÓN}

\section{CARACTERIZACIÓN DE LOS RESIDUOS ORGÁNICOS AVÍCOLAS}

1.1. Análisis elemental

La caracterización de la gallinaza (GG), pollinaza (CP) y gallinaza-pollinaza (GG-CP) se recogen en la tabla 2 . 
494 Enmer Trujillo A., Christian Eduardo Valencia A., María Cecilia Alegría A., Alejandrina Honorata Sotelo M...

Tabla 2. Caracterización de los residuos avícolas de análisis elemental.

\begin{tabular}{ccccccc}
\hline Biomasa & $\mathbf{C}(\%)$ & $\mathbf{N}(\%)$ & $\mathbf{C} / \mathbf{N}$ & Ceniza (\%) & Humedad (\%) & $\mathbf{p H}$ \\
\hline Gallinaza (GG) & 17,61 & 1,90 & 9,27 & 62,80 & 10,63 & 8,62 \\
Pollinaza (PO) & 24,53 & 2,48 & 9,89 & 25,19 & 18,84 & 8,74 \\
(GG-PO) & 16,00 & 2,37 & 6,75 & 37,38 & 15,44 & 8,51 \\
\hline
\end{tabular}

Fuente: Elaboración propia

Del análisis químico elemental, el contenido del carbono orgánico promedio obtenido es mayor en la pollinaza $(24,53 \%)$ que en la gallinaza $(17,61 \%)$, lo cual puede corroborarse con los resultados cercanos obtenidos por otro estudio ${ }^{12}$, siendo estos de 23,0 y $19,8 \%$ de carbón, respectivamente; sin embargo, otro autor ${ }^{13}$ reporta un valor de $31,71 \%$ para gallinaza de jaula y de $38,70 \%$ para la gallinaza de piso, siendo estos valores mayores al obtenido, así también se observa que la mezcla de ambos residuos avícolas presenta menor porcentaje $(16,0 \%)$.

El contenido de nitrógeno obtenido fue mayor para la pollinaza $(2,48 \%)$ que para la gallinaza $(1,90 \%)$. Otro autor ${ }^{4}$ reportó valores cercanos $(2,3 \%)$ para la pollinaza, sin embargo el autor ${ }^{14}$ reportó para la gallinaza 3,24\%, siendo este valor casi el doble del valor obtenido. Así, la relación carbono y nitrógeno $(\mathrm{C} / \mathrm{N})$ obtenido está entre $(9,27-9,89)$ para la gallinaza y pollinaza, respectivamente, valor muy cercano al encontrado por otros autores ${ }^{4,13}$ para la pollinaza 9,7 y 9,79, respectivamente, sin embargo, otro autor ${ }^{4}$ reporta un valor un poco alto para la gallinaza $(12,02)$, así1 ${ }^{14}$, reporta una pequeña variación $(9,79)$, se observa sin embargo que la mezcla de estos dieron menores resultados $(6,75)$.

Con respecto al porcentaje de cenizas obtenidas, la gallinaza reporta un valor bastante alto $(62,8 \%)$, casi el doble con respecto al de la pollinaza $(25,19 \%)$, estos resultados se han corroborado $^{15}$, siendo estos valores 28 y $15 \%$ para la gallinaza y pollinaza, respectivamente.

La humedad reportada para la gallinaza es de $10,63 \%$ y para la pollinaza de $18,84 \%$, sin embargo, otro autor ${ }^{14}$ ha reportado para la gallinaza $(26,08 \%)$ siendo este valor mayor al doble del obtenido, así otros autores ${ }^{15}$ han obtenido $23,14 \%$ de humedad para muestras secas, siendo este valor no muy lejano al obtenido. Estos resultados, tanto para la gallinaza y pollinaza, se deben a que las muestras usadas para este estudio fueron secadas previamente a temperatura ambiente.

El pH de estos residuos avícolas varía entre 8,51-8,74 en la escala fuertemente alcalina, este intervalo se encuentra dentro de los valores reportados ${ }^{13}$, encontrándose estos en un intervalo de (8-9), otro autor ${ }^{4}$ reportó valores un poco más altos de $\mathrm{pH}$, siendo estos valores de 9,0 y 9,5 para la gallinaza y pollinaza, respectivamente. 


\subsection{Análisis de nutrientes}

Los resultados del contenido de macro y micronutrientes como se observa en el análisis inmediato en la tabla 3.

Tabla 3. Caracterización química de los residuos avícolas.

\begin{tabular}{|c|c|c|c|c|c|c|c|c|c|c|}
\hline Biomasa & $\begin{array}{c}\mathbf{N} \\
(\mathrm{g} / 100 \mathrm{~g})\end{array}$ & $\begin{array}{c}\mathbf{P} \\
(\mathrm{mg} / \mathrm{kg})\end{array}$ & $\begin{array}{c}\mathbf{K} \\
(\mathrm{mg} / \mathrm{kg})\end{array}$ & $\begin{array}{c}\mathbf{N a} \\
(\mathrm{mg} / \mathrm{kg})\end{array}$ & $\begin{array}{c}\text { Ca } \\
(\mathrm{mg} / \mathrm{kg})\end{array}$ & $\begin{array}{c}\mathbf{C u} \\
(\mathrm{mg} / \mathrm{kg})\end{array}$ & $\begin{array}{c}\mathbf{F e} \\
(\mathrm{mg} / \mathrm{kg})\end{array}$ & $\begin{array}{c}\text { Mg } \\
(\mathrm{mg} / \mathrm{kg})\end{array}$ & $\begin{array}{c}\text { Mn } \\
(\mathrm{mg} / \mathrm{kg})\end{array}$ & $\begin{array}{c}\mathbf{Z n} \\
(\mathrm{mg} / \mathrm{kg})\end{array}$ \\
\hline $\begin{array}{l}\text { Gallinaza } \\
\text { (GG) }\end{array}$ & 1,90 & 20578,72 & 34835,73 & 7920,46 & 269822,48 & 56,51 & 6363,08 & 87539,58 & 493,95 & 425,53 \\
\hline $\begin{array}{l}\text { Pollinaza } \\
\text { (PO) }\end{array}$ & 2,48 & 12882,65 & 12470,29 & 7208,20 & 27217,44 & 42,82 & 1770,82 & 30040,69 & 364,56 & 380,38 \\
\hline (GG-PO) & 2,37 & 18312,17 & 24894,81 & 8512,91 & 74349,30 & 55,02 & 3371,57 & 58580,32 & 423,49 & 421,83 \\
\hline
\end{tabular}

Se evidencia que el contenido del nitrógeno de la pollinaza $(2,48)$ es mayor que la gallinaza $(1,90)$ y para el contenido de la mezcla $(2,37)$. Así, el contenido de macronutrientes $(\mathrm{P}, \mathrm{K}, \mathrm{Na}$, $\mathrm{Ca}$ y $\mathrm{Mg}$ ) de la gallinaza presenta valores mucho mayores a los observados para la pollinaza, a excepción de nitrógeno, el cual es menor. En el caso del contenido de micronutrientes (Fe, $\mathrm{Cu}, \mathrm{Mn}$ y $\mathrm{Zn}$ ) de la gallinaza, estos presentan valores mayores con respecto a la pollinaza, siendo el contenido de fierro casi seis veces mayor al de la pollinaza, estos resultados se deben a que las camas de los pollos de engorde son menos ricos en nutrientes que las de las gallinas.

\section{CARACTERIZACIÓN DEL BIOCHAR}

\subsection{Análisis físico elemental}

Se encontró una diferencia significativa ( $\mathrm{p}$-valor $<0,05)$ entre la media de rendimientos de los tratamientos, se puede concluir que la media de los rendimientos del biochar de la gallinaza y pollinaza es muy significativa. El rendimiento medio de producción del biocarbón de la gallinaza es de $64,31 \%$, mientras de la pollinaza fue de $42,99 \%$, pero el rendimiento medio de la mezcla entre ellos es 55,14\%.

Se encontró una diferencia muy significativa ( $\mathrm{p}$-valor $<0,05)$ entre la media de la densidad aparente de la gallinaza y pollinaza. Se observa para gallinaza una densidad aparente entre $0,564-0,580 \mathrm{~g} / \mathrm{cm}^{3}$ e intervalos de $0,330-0,341 \mathrm{~g} / \mathrm{cm}^{3}$ para la pollinaza. Los resultados de la densidad aparente de pollinaza están dentro de los límites óptimos recomendables por reducir notablemente el volumen original hasta del 61,52 \%, mientras la disminución del volumen original para la gallinaza es de 56,26 \% lo cual tiene una enorme ventaja en la conversión en biochar, los residuos avícolas en términos de volumen serían una buena alternativa de manejo y gestión.

Las características morfológicas y estructurales resultantes del biochar de la gallinaza y pollinaza son como se muestra en las imágenes de la figura 2. 

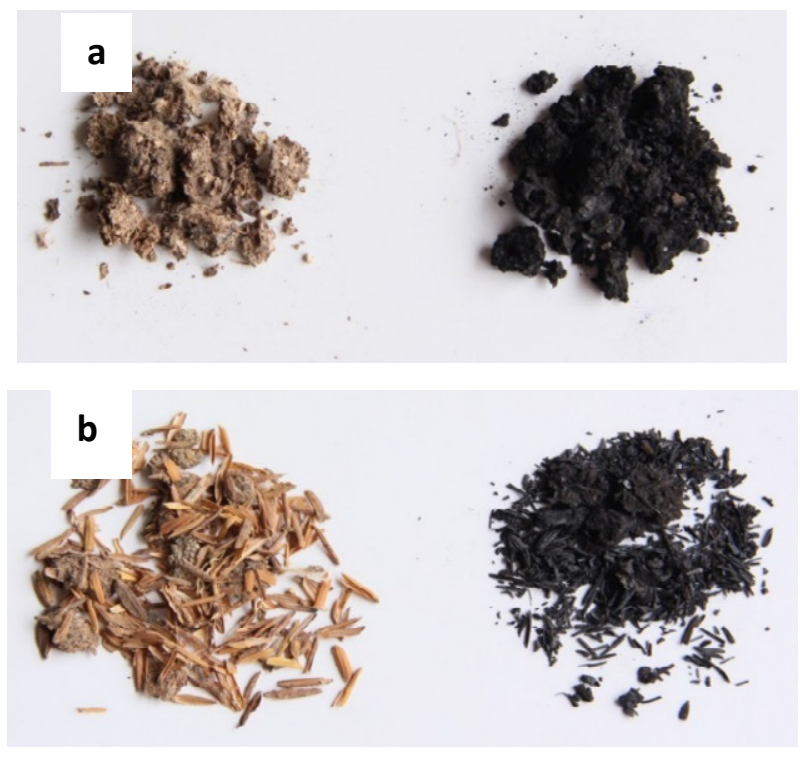

Figura 2. Muestras representativas de biomasa residual y respectivo biochar resultante. a) Muestra para gallinaza, b) Muestra para pollinaza.

En la tabla 4 se detallan el tiempo y promedio de temperatura de pirólisis empleado, porcentaje de rendimientos y densidad del biochar por cada tratamiento y sus respectivas repeticiones.

Tabla 5. Resultados del análisis físico elemental del biochar.

\begin{tabular}{|c|c|c|c|c|c|c|c|}
\hline BIOCHAR & REP & $\begin{array}{l}\text { BIOMASA } \\
\text { (g) }\end{array}$ & $\begin{array}{c}\text { BIOCHAR } \\
\text { RESULTANTE } \\
\text { (g) }\end{array}$ & $\begin{array}{l}\text { RENDIMIENTO } \\
\text { BIOCHAR (\%) }\end{array}$ & $\begin{array}{l}\text { DENSIDAD } \\
\text { BIOCHAR } \\
\mathrm{g} / \mathrm{cm}^{3}\end{array}$ & $\begin{array}{l}\text { PROM. } \\
\text { T }^{\circ} \mathrm{C}\end{array}$ & $\begin{array}{c}\text { TIEMPO } \\
\text { PROM. } \\
\text { PIRÓLISIS } \\
\text { Hrs. }\end{array}$ \\
\hline \multirow{3}{*}{$\begin{array}{l}\text { GALLINAZA } \\
\text { (GG) }\end{array}$} & $\mathrm{R} 1$ & 4000 & 2567 & 64,18 & 0,567 & 556 & 3 \\
\hline & $\mathrm{R} 2$ & 4000 & 2578 & 64,45 & 0,58 & 545 & 3 \\
\hline & R3 & 4000 & 2572 & 64,3 & 0,564 & 549 & 3 \\
\hline \multirow{3}{*}{$\begin{array}{l}\text { POLLINAZA } \\
\text { (CP) }\end{array}$} & $\mathrm{R} 1$ & 3000 & 1291 & 43,03 & 0,333 & 529 & 3 \\
\hline & $\mathrm{R} 2$ & 3000 & 1285 & 42,83 & 0,341 & 519 & 3 \\
\hline & R3 & 3000 & 1294 & 43,13 & 0,33 & 523 & 3 \\
\hline \multirow{3}{*}{ GG-CP } & $\mathrm{R} 1$ & 3000 & 1649 & 54,97 & 0,387 & 539 & 3 \\
\hline & $\mathrm{R} 2$ & 3000 & 1654 & 55,13 & 0,385 & 544 & 3 \\
\hline & R3 & 3000 & 1660 & 55,33 & 0,383 & 550 & 3 \\
\hline
\end{tabular}

\subsection{Análisis químico elemental}

En la tabla 5 se presentan los resultados obtenidos del análisis químico elemental para las muestras de biochar de gallinaza, pollinaza y la mezcla de ambos. 
Tabla 5. Análisis químico elemental del biochar resultante en relación a la materia prima original.

\begin{tabular}{clccccc}
\hline BIOCHAR & Rep & $\begin{array}{c}\mathbf{C} \\
(\mathbf{g} / \mathbf{1 0 0} \mathbf{g})\end{array}$ & $\mathbf{C} / \mathbf{N}$ & $\begin{array}{c}\text { Ceniza } \\
(\mathrm{g} / \mathbf{1 0 0} \mathbf{g})\end{array}$ & $\begin{array}{c}\text { Humedad } \\
\text { (\%) }\end{array}$ & $\mathbf{p H}$ \\
\hline \multirow{2}{*}{ Gallinaza } & R0 & 17,61 & 9,27 & 62,8 & 10,63 & 8,62 \\
(GG) & R1 & $\mathbf{8 , 4 7}$ & $\mathbf{7 , 9 2}$ & $\mathbf{8 0 , 3 6}$ & $\mathbf{0 , 9 5}$ & $\mathbf{1 0 , 2 3}$ \\
& R2 & $\mathbf{7 , 8 5}$ & $\mathbf{7 , 2 7}$ & $\mathbf{8 1 , 8 4}$ & $\mathbf{0 , 5 9}$ & $\mathbf{1 0 , 2 1}$ \\
& R3 & $\mathbf{8 , 4 7}$ & $\mathbf{8 , 4 7}$ & $\mathbf{8 3 , 8 6}$ & $\mathbf{0 , 5 7}$ & $\mathbf{1 0 , 0 2}$ \\
Pollinaza & R0 & 24,53 & 9,89 & 25,19 & 18,84 & 8,74 \\
(PO) & R1 & $\mathbf{9 , 5 3}$ & $\mathbf{4 , 8 4}$ & $\mathbf{5 5 , 2}$ & $\mathbf{1 , 5 7}$ & $\mathbf{1 0 , 6 8}$ \\
& R2 & $\mathbf{1 0 , 8 3}$ & $\mathbf{4 , 7 7}$ & $\mathbf{5 2 , 2 9}$ & $\mathbf{1 , 8 9}$ & $\mathbf{1 0 , 9 8}$ \\
& R3 & $\mathbf{8 , 0 7}$ & $\mathbf{4 , 2 3}$ & $\mathbf{5 6 , 4 7}$ & $\mathbf{1 , 9 3}$ & $\mathbf{1 1 , 0 1}$ \\
& R0 & 16 & 6,75 & 37,38 & 15,44 & 8,51 \\
(GG-PO) & R1 & $\mathbf{2 , 7 1}$ & $\mathbf{1 , 9}$ & $\mathbf{7 1 , 5 3}$ & $\mathbf{1 , 2 7}$ & $\mathbf{1 0 , 1 8}$ \\
& R2 & $\mathbf{6 , 6 7}$ & $\mathbf{5 , 1 7}$ & $\mathbf{7 1 , 6 5}$ & $\mathbf{1 , 5 9}$ & $\mathbf{9 , 7 3}$ \\
& R3 & $\mathbf{6 , 4 4}$ & $\mathbf{4 , 9 2}$ & $\mathbf{6 9 , 2 8}$ & $\mathbf{1 , 3 3}$ & $\mathbf{1 0 , 1 2}$ \\
\hline
\end{tabular}

Fuente: La Molina Calidad Total Laboratorios

\section{Carbono orgánico total:}

El análisis estadístico muestra una diferencia no significativa ( $p$-valor $>0,5$ ) entre el contenido de carbono de gallinaza, pollinaza y la mezcla. Los resultados promedios del carbono orgánico total del biochar de gallinaza fue $8,26 \mathrm{~g} / 100 \mathrm{~g}$, la pollinaza 9,48 g/100g, la mezcla contiene un resultado inferior de $5,27 \mathrm{~g} / 100 \mathrm{~g}$. Se observa que la materia prima original de mayor concentración como la pollinaza $(24,53)$, genera una concentración media mayor de carbono orgánico total de $9,38 \mathrm{~g} / 100 \mathrm{~g}$. Todos estos datos son inferiores al contenido de carbono total en camas de pollo $(42,9 \mathrm{~g} / \mathrm{kg})^{14}$. Este parámetro es muy importante para el biochar por la fijación del carbón en el suelo lo cual le confiere estabilidad por periodos largos, así como la cantidad y disponibilidad de nutrientes en el suelo ${ }^{3}$, otro autor ${ }^{16}$ indica mayores contenidos de carbono al obtenido, siendo este entre intervalos 29 a 46,10 g/100 g. de biochar para rangos de temperatura de 350 a $770^{\circ} \mathrm{C}$.

\section{Relación C/N:}

Los resultados de la relación $\mathrm{C} / \mathrm{N}$ de los biocarbones es significativa entre la gallinaza $(7,88)$ y pollinaza $(4,61)$, mientras la mezcla gallinaza - pollinaza resultó una media inferior $(3,99)$. Estos resultados son inferiores a $(13,0)^{14}$ obtenidos en biocarbones de cama de pollo ${ }^{17}$. Otro autor ${ }^{18}$ indica una relación $\mathrm{C} / \mathrm{N}$ entre 7 - 400 para biocarbones de restos avícolas, la relación $\mathrm{C} / \mathrm{N}$ es un parámetro importante, ya que indica la habilidad de un sustrato orgánico de mineralizar e introducir nitrógeno inorgánico al suelo, un valor alto podría causar inmovilización del nitrógeno inorgánico por los microorganismos causando deficiencia de nitrógeno para el crecimiento de plantas. El resultado obtenido es bajo al comparar la relación $\mathrm{C} / \mathrm{N}$ con el requerimiento para compost (25 - 30)1. 
498 Enmer Trujillo A., Christian Eduardo Valencia A., María Cecilia Alegría A., Alejandrina Honorata Sotelo M...

\section{Valores de pH:}

Hay una diferencia muy significativa ( $\mathrm{p}$-valor $<0,05$ ) entre la media de valores de $\mathrm{pH}$ de biochar de gallinaza y pollinaza, pero con resultados similares entre el biochar de la gallinaza y la mezcla gallinaza - pollinaza. Los valores media de $\mathrm{pH}$ del biochar gallinaza fue 10,15, mientras la pollinaza tiene una media alta de 10,89 y la mezcla contiene valor medio inferior $(10,01)$. Los datos revelan que los valores del $\mathrm{pH}$ de la gallinaza y pollinaza son muy fuertemente alcalinos, así como las correspondientes mezclas. Estos valores son similares ${ }^{14}$, quienes observaron el valor de $\mathrm{pH}(10,2)$ muy alcalinos en camas de pollo. Así, otro autor ${ }^{19}$ indica que la mayoría de los biochars producidos a pirólisis lenta puede ir de 7,5 a 10,5, es decir de medianamente alcalinos a fuertemente alcalinos a altas temperaturas.

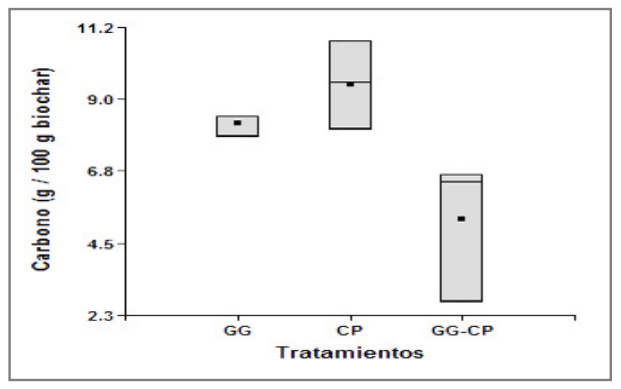

Figura 3. Carbono orgánico total.

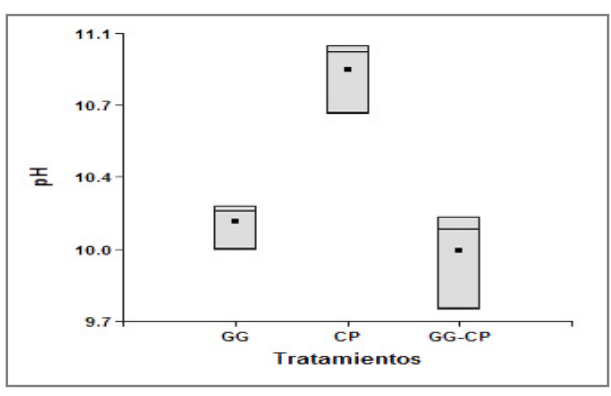

Figura 4. Valores del pH.

\subsection{Análisis proximal}

\section{Humedad:}

Presenta una diferencia significativa ( $\mathrm{p}$-valor $<0,05)$ entre la media de humedad de biochar de gallinaza y pollinaza. El contenido de humedad en los biocarbones de la gallinaza tuvo una concentración media de $0,70 \%$, en la pollinaza resulta una concentración mayor de $1,80 \%$ y en la mezcla gallinaza - pollinaza de 1,40\%. La cantidad de humedad presente en el biochar dependerá del incremento de la temperatura en el proceso de la pirólisis y también del método usado para su determinación que en la presente investigación se usó la norma peruana NTP 311.0121970 .

\section{Cenizas:}

El contenido de cenizas del biochar muestra una diferencia significativa ( $p$-valor $<0,05$ ) entre la media de los tratamientos. El contenido de cenizas en el biochar de los residuos orgánicos depende del contenido de cenizas de la biomasa de origen. El contenido medio de cenizas del biochar resulta alto en el biochar de la gallinaza $(82,02 \mathrm{~g} / 100 \mathrm{~g})$ mientras de la pollinaza tuvo una media inferior $(54,65 \mathrm{~g} / 100 \mathrm{~g})$. Se observa que el contenido de cenizas de biochar de pollinaza son superiores a los resultados reportados por otro autor ${ }^{38}$ de $30,7 \%$ y $46,2 \%$ en peso a conversión térmica de $350^{\circ} \mathrm{C}$ y $700^{\circ} \mathrm{C}$, respectivamente. 


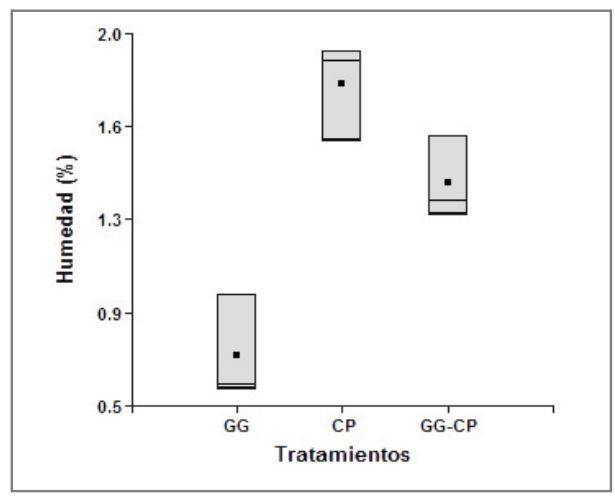

Figura 5. Humedad del biochar.

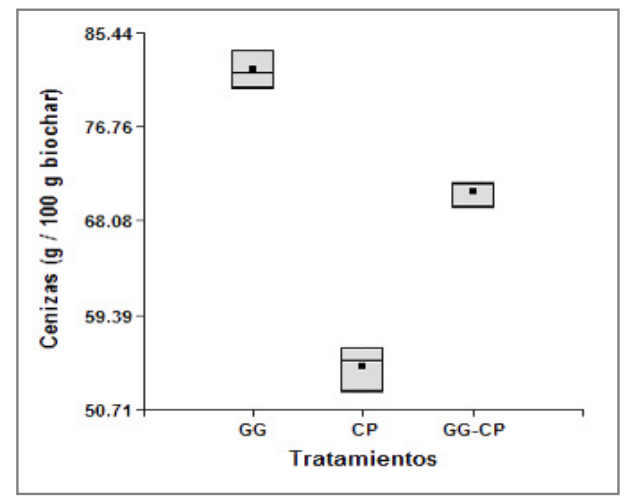

Figura 6. Cenizas del biochar.

\subsection{Análisis de nutrientes}

\subsubsection{Micronutrientes}

El análisis estadístico del contenido de micronutrientes del biochar, tanto de gallinaza y pollinaza, muestran una diferencia significativa ( $\mathrm{p}$-valor $<0,05$ ), lo cual se ve reflejada en los resultados promedios (tabla 6) del contenido de cobre, hierro, manganeso y zinc, los cuales son mayores comparados con la materia prima original, para el biochar de pollinaza se observa contenidos de cobre, manganeso y zinc casi al doble del contenido del material original, sin embargo el contenido de hierro es cinco veces mayor al contenido de micronutrientes de la materia prima original. Los resultados del biochar de la mezcla muestran resultados casi al doble del contenido de cobre, hierro, manganeso y zinc, con respecto a la materia prima original.

\subsubsection{Macronutrientes}

Para los macronutrientes el análisis estadístico muestra una diferencia significativa ( $\mathrm{p}$-valor $<0,05$ ) lo cual se evidencia en la tabla 6 , esta muestra los resultados promedios tanto para el biochar de gallinaza, pollinaza y de la mezcla de ambas. Se evidencia que el contenido de nitrógeno es un poco menor para el biochar de gallinaza $(1,29 \mathrm{mg} / \mathrm{kg})$ y la mezcla de ambas $(1,6 \mathrm{mg} / \mathrm{kg})$, con respecto al contenido del material original gallinaza $(1,9 \mathrm{mg} / \mathrm{kg})$ y de la mezcla de ambas $(2,37 \mathrm{mg} / \mathrm{kg})$, sin embargo, el contenido de calcio $(203360,84)$ en el biochar de gallinaza es bastante menor al del material original $(269822,48 \mathrm{mg} / \mathrm{kg})$, así como el contenido de sodio en el biochar de pollinaza $(5898,39)$ es menor con respecto a la materia prima $(7208,2 \mathrm{mg} / \mathrm{kg})$. Mientras que el contenido de fósforo en el biochar de pollinaza $(25270,92 \mathrm{mg} / \mathrm{kg})$ y el contenido de potasio en el biochar de la mezcla $(48807,23$ $\mathrm{mg} / \mathrm{kg}$ ) es el doble al de la materia prima $(12882,65,24894,81) \mathrm{mg} / \mathrm{kg}$ de fósforo y potasio, respectivamente, para el caso del biochar de gallinaza el contenido de potasio es casi el doble al del material original. 
500 Enmer Trujillo A., Christian Eduardo Valencia A., María Cecilia Alegría A., Alejandrina Honorata Sotelo M...

Tabla 6. Análisis del contenido de nutrientes del biochar a partir de los residuos avícolas (R.A).

\begin{tabular}{|c|c|c|c|c|c|c|c|c|c|c|c|}
\hline BIO & Rep & $\underset{(\mathrm{mg} / \mathrm{kg})}{\mathrm{N}}$ & $\underset{(\mathrm{mg} / \mathrm{kg})}{\mathbf{P}}$ & $\underset{(\mathrm{mg} / \mathrm{kg})}{\mathrm{K}}$ & $\underset{(\mathrm{mg} / \mathrm{kg})}{\mathrm{Na}}$ & $\underset{(\mathrm{mg} / \mathrm{kg})}{\mathrm{Ca}}$ & $\underset{(\mathrm{mg} / \mathrm{kg})}{\mathrm{Cu}}$ & $\begin{array}{c}\mathrm{Fe} \\
(\mathrm{mg} / \mathrm{kg})\end{array}$ & $\underset{(\mathbf{m g} / \mathbf{k g})}{\mathbf{M g}}$ & $\underset{(\mathrm{mg} / \mathrm{kg})}{\mathbf{M n}}$ & $\underset{(\mathrm{mg} / \mathrm{kg})}{\mathrm{Zn}}$ \\
\hline \multirow{5}{*}{$\begin{array}{l}\text { Gallinaza } \\
\text { (GG) }\end{array}$} & R0 & 1,9 & 20578,72 & 34835,73 & 7920,46 & 269822,48 & 56,51 & 6363,08 & 87539,58 & 493,95 & 425,53 \\
\hline & R1 & 1,07 & 36232,88 & 68664,52 & 784,43 & 168798,73 & 101,94 & 6820,06 & 94776,18 & 852,97 & 741,82 \\
\hline & $\mathbf{R} 2$ & 1,08 & 36202,59 & 57554,02 & 14809,57 & 218355,81 & 94,92 & 8097,47 & 99560,02 & 825,12 & 717,83 \\
\hline & R3 & 1 & 30211,8 & 44683,31 & 13750,12 & 156466,34 & 91,18 & 7910,44 & 89293,98 & 735,1 & 617,36 \\
\hline & Prom & 1,26 & 30806,50 & 51434,40 & 9316,15 & 203360,84 & 86,14 & 7297,76 & 92792,44 & 726,79 & 625,64 \\
\hline \multirow{4}{*}{$\begin{array}{l}\text { Pollinaza } \\
\text { (PO) }\end{array}$} & R0 & 2,48 & 12882,65 & 12470,29 & 7208,2 & 27217,44 & 42,82 & 1770,82 & 30040,69 & 364,56 & 380,38 \\
\hline & R1 & 1,97 & 30128,04 & 71165,96 & 764,05 & 64704,42 & 103,14 & 4878,31 & 69302,7 & 925,81 & 706,13 \\
\hline & R2 & 2,27 & 29454,86 & 63095,44 & 14852,05 & 56112,77 & 72,88 & 3755,6 & 63722,21 & 832,86 & 628,33 \\
\hline & R3 & 1,91 & 28618,14 & 61839,87 & 769,25 & 55236,33 & 75,83 & 5552,68 & 61578,07 & 769,25 & 564,6 \\
\hline \multirow{6}{*}{ (GG-PO) } & Prom & 2,16 & 25270,92 & 52142,89 & 5898,39 & 50817,74 & 73,67 & 3989,35 & 56160,92 & 723,12 & 569,86 \\
\hline & R0 & 2,37 & 18312,17 & 24894,81 & 8512,91 & 74349,3 & 55,02 & 3371,57 & 58580,32 & 423,49 & 421,83 \\
\hline & R1 & 1,43 & 29202,75 & 57816,37 & 14294,83 & 238768,75 & 93,75 & 6193,56 & 97784,22 & 756,6 & 714,87 \\
\hline & R2 & 1,29 & 31553,87 & 59907,88 & 15686,71 & 259249,4 & 103,44 & 5413,49 & 92284,84 & 780,15 & 714,31 \\
\hline & R3 & 1,31 & 27083,38 & 52609,86 & 9707,99 & 304813,26 & 100,29 & 5919,38 & 99263,69 & 735,64 & 705,72 \\
\hline & Prom & 1,6 & 26538,0425 & 48807,23 & 12050,61 & 219295,18 & 88,13 & 5224,50 & 86978,27 & 673,97 & 639,18 \\
\hline
\end{tabular}

Fuente: Laboratorio de Análisis de Suelos, Plantas, Aguas y Fertilizantes

\section{ANÁLISIS DE BIOENSAYOS}

Los análisis de bioensayos se muestran en la tabla 7 y la figura 7, estos presentan los resultados de los bioensayos y se evidencia la media del porcentaje de índice de germinación (IG) como producto del porcentaje de germinación relativa (PGR) y el crecimiento del tallo relativo (CTR). Para ello se siguió la metodología del test para la determinación del Índice de Germinación (IG) que permitió determinar la media del porcentaje de germinación de las semillas y el crecimiento de las plántulas o tallos 9 .

Tabla 7. Resumen de indicadores de bioensayos de toxicidad de los biocarbones.

\begin{tabular}{ccccccc}
\hline $\begin{array}{c}\text { Trats. } \\
\text { (\%) }\end{array}$ & PGR & $\begin{array}{c}\text { Gallinaza (GG) } \\
\text { CTR }\end{array}$ & IG (\%) & PGR & $\begin{array}{c}\text { Pollinaza (CP) } \\
\text { CTR }\end{array}$ & IG (\%) \\
\hline \multirow{4}{*}{1} & 70 & 109,9 & 76,93 & 100 & 61,2 & 61,2 \\
& 60 & 64,2 & 38,52 & 90 & 65,6 & 59,04 \\
& 80 & 101 & 80,8 & 90 & 75,8 & 68,22 \\
& $\mathbf{7 0}$ & $\mathbf{9 1 , 7}$ & $\mathbf{6 5 , 4 2}$ & $\mathbf{9 3 , 3 3}$ & $\mathbf{6 7 , 5 3}$ & $\mathbf{6 2 , 8 2}$ \\
& 80 & 91,1 & 72,88 & 80 & 62,6 & 50,08 \\
$\mathbf{3}$ & 90 & 85,8 & 77,22 & 90 & 33,1 & 29,79 \\
& 90 & 100,1 & 90,09 & 80 & 43,8 & 35,04 \\
& $\mathbf{8 6 , 6 7}$ & $\mathbf{9 2 , 3 3}$ & $\mathbf{8 0 , 0 6}$ & $\mathbf{8 3 , 3 3}$ & $\mathbf{4 6 , 5}$ & $\mathbf{3 8 , 3}$ \\
& 80 & 75,6 & 60,48 & 60 & 18,8 & 11,28 \\
$\mathbf{5}$ & 80 & 93,3 & 74,64 & 50 & 22,6 & 11,3 \\
& 80 & 87,4 & 69,92 & 60 & 45,2 & 27,12 \\
& $\mathbf{8 0}$ & $\mathbf{8 5 , 4 3}$ & $\mathbf{6 8 , 3 5}$ & $\mathbf{5 6 , 6 7}$ & $\mathbf{2 8 , 8 7}$ & $\mathbf{1 6 , 5 7}$ \\
& 100 & 43,8 & 43,8 & 30 & 19 & 5,7 \\
$\mathbf{7}$ & 100 & 72,3 & 72,3 & 50 & 12,6 & 6,3 \\
& 70 & 83,1 & 58,17 & 50 & 9,2 & 4,6 \\
& $\mathbf{9 0}$ & $\mathbf{6 6 , 4}$ & $\mathbf{5 8 , 0 9}$ & $\mathbf{4 3 , 3 3}$ & $\mathbf{1 3 , 6}$ & $\mathbf{5 , 5 3}$ \\
\hline
\end{tabular}

PGR: Porcentaje de germinación relativa

CTR: Crecimiento del tallo relativo

IG (\%): Porcentaje de índice de germinación 


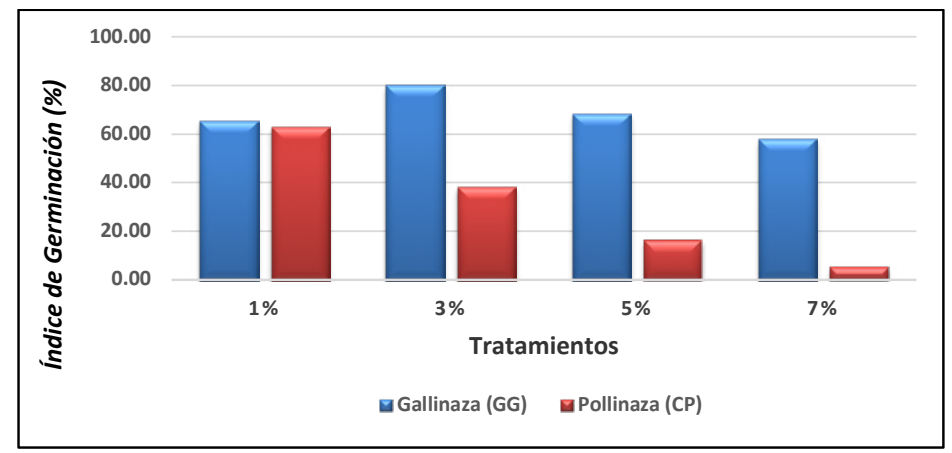

Figura 7. Índice de germinación en los cuatro tratamientos de biocarbones.

Los resultados del índice de germinación (IG) de las semillas de rabanito con la gallinaza fue superior $(80,06 \%)$ en tratamiento del $3 \%$, mientras el tratamiento al $1 \%$ de biochar de pollinaza produjo el IG de $62,82 \%$. Se concluye que los rabanitos son especies que son sensibles a sustancias fitotóxicas. Los biochars de origen de la gallinaza en tratamientos de $1 \%$ a $7 \%$ indican presencias moderadas de sustancias tóxicas. Pero los biochar de origen de la pollinaza en concentraciones superiores al $3 \%$ inhiben la germinación de las semillas por la fuerte presencia de concentraciones de sustancias tóxicas.

En la tabla 8 y las figuras 8 y 9 se presentan los resultados del peso fresco de los diez primeros días de plántulas de semillas de rabanito de los cuatro tratamientos.

Tabla 8. Crecimiento de tallo y peso fresco de plántulas de semillas de rabanito.

\begin{tabular}{|c|c|c|c|c|}
\hline \multirow{2}{*}{$\begin{array}{l}\text { Trats. } \\
\text { (\%) }\end{array}$} & \multicolumn{2}{|c|}{ Crecimiento de tallo (cm) } & \multicolumn{2}{|c|}{ Peso de biomasa fresco $(\mathrm{g})$} \\
\hline & $\begin{array}{c}\text { Gallinaza } \\
\text { (GG) }\end{array}$ & $\begin{array}{c}\text { Pollinaza } \\
(\mathrm{CP})\end{array}$ & $\begin{array}{c}\text { Gallinaza } \\
\text { (GG) }\end{array}$ & $\begin{array}{l}\text { Pollinaza } \\
\text { (CP) }\end{array}$ \\
\hline \multirow{4}{*}{1} & 10,99 & 6,12 & 0,934 & 0,6993 \\
\hline & 6,42 & 6,56 & 0,4618 & 0,6455 \\
\hline & 10,1 & 7,58 & 0,8429 & 0,8116 \\
\hline & 9,17 & 6,75 & 0,7462 & 0,7188 \\
\hline \multirow{4}{*}{3} & 9,11 & 6,26 & 0,679 & 0,5227 \\
\hline & 8,58 & 3,31 & 0,7983 & 0,4464 \\
\hline & 10,01 & 4,38 & 0,8027 & 0,4138 \\
\hline & 9,23 & 4,65 & 0,76 & 0,461 \\
\hline \multirow{4}{*}{5} & 7,56 & 1,88 & 0,6032 & 0,2426 \\
\hline & 9,33 & 2,26 & 0,798 & 0,2107 \\
\hline & 8,74 & 4,52 & 0,8024 & 0,4394 \\
\hline & 8,54 & 2,89 & 0,7345 & 0,2976 \\
\hline \multirow{4}{*}{7} & 4,38 & 1,9 & 0,5444 & 0,1008 \\
\hline & 7,23 & 1,26 & 0,8048 & 0,1306 \\
\hline & 8,31 & 0,92 & 0,6052 & 0,1738 \\
\hline & 6,64 & 1,36 & 0,6515 & 0,1351 \\
\hline
\end{tabular}




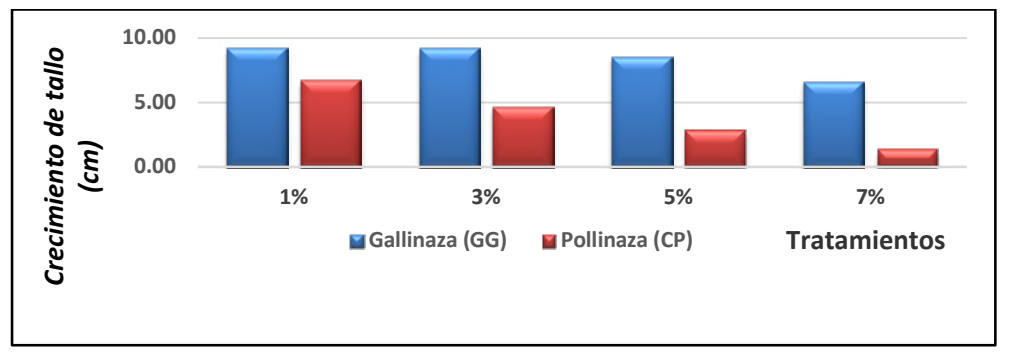

Figura 8. Crecimiento de tallo de plántulas con diferentes tratamientos de biochar.

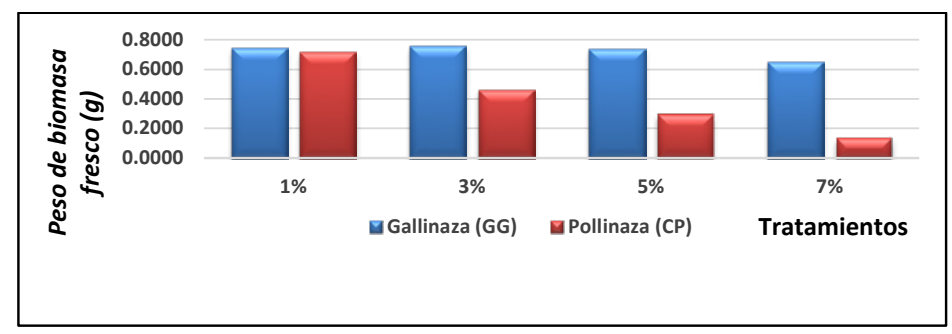

Figura 9. Peso fresco de la longitud del crecimiento de las plántulas por tratamientos.

El crecimiento de las plántulas con el biochar de gallinaza (GG) fueron mayores para los tratamientos de $1 \%$ y $3 \%$. Los tratamientos con el biochar de la pollinaza $(\mathrm{CP})$ demostraron un mejor crecimiento de las plántulas con el $1 \%$. En base a la evidencia de las medias del peso fresco de las plántulas de las semillas de rabanito, el tratamiento al $3 \%$ de biochar de la gallinaza $(\mathrm{GG})$ presentó un peso fresco mayor $(0,7600 \mathrm{~g})$. Mientras los biochar de la pollinaza $(\mathrm{CP})$ aplicados en tratamientos al $1 \%$, presentaron una media de peso fresco $(0,7188 \mathrm{~g})$ mayor que al $3 \%, 5 \%$ y $7 \%$.

\section{CONCLUSIONES}

La producción de biochar de gallinaza presenta mayores rendimientos promedio de $64,31 \%$ a diferencia del biochar de pollinaza $43,00 \%$, siendo los rendimientos de estos mayores al obtenido de biocarbones de biomasa lignocelulósica $(27 \%)^{21}$.

La caracterización química del biochar, en particular de la pollinaza, presenta valores óptimos de densidad aparente, con la consecuente reducción en volumen de la materia prima original. Se puede lograr a ocupar menor espacio de almacenamiento, una característica beneficiosa de manejo, transporte y comercialización de los residuos. Asimismo, se observa valores altos de $\mathrm{pH}(10,23)$ en ambos biocarbones, lo cual indica que podría usarse para la recuperación de suelos ácidos característicos de la selva. 
El índice de germinación, como variable que integra diferentes grados de fitotoxicidad, es más elevado en los biocarbones de origen de la gallinaza que en la de la pollinaza al $3 \%$. La adición de los biocarbones de los residuos avícolas, en particular de la gallinaza, aumenta considerablemente el crecimiento de las semillas y los pesos frescos de las plántulas a concentraciones de $1 \%$ y $3 \%$ y una adición superior a estos porcentajes provocan efectos negativos con mayor evidencia en los biocarbones de la pollinaza.

\section{AGRADECIMIENTO}

Al Vicerrectorado de Investigación de la Universidad Nacional Agraria La Molina, por el financiamiento (concurso de Innovación Tecnológica IT - UNALM - 2016) del proyecto de investigación "Producción y caracterización de biochar vegetal a partir de residuos orgánicos avícolas y biomasa residual de la podadura de áreas verdes".

\section{REFERENCIAS BIBLIOGRÁFICAS}

1. García C, Rosas JG, Sánchez ME, Pascual JE, Hernández MT. Enmiendas orgánicas de nueva generación: Biochar y otras biomoléculas. Madrid: Editorial Mundi-Prensa; 2014.

2. MINAN DL N ${ }^{\circ}$ 1278. Ley de Gestión Integral de Residuos Sólidos. El Peruano [Internet]. 2016. [Consultado 4 jun 2019]. Disponible en: http://www.minam.gob.pe/disposiciones/ decreto-legislativo-n-1278/

3. Escalante A, Pérez G, Hidalgo C, López J, Campo J, Valtierra E, Etchevers J. Biocarbón (biochar) I: Naturaleza, historia, fabricación y uso en el suelo. Sociedad Mexicana de la Ciencia del Suelo. A.C. México. Terra Latinoamericana. 2016; 34(3): 367-382.

4. Estrada MM. Manejo y Procesamiento de la gallinaza. Corporación Universitaria Lasallista. Antioquia, Colombia. Revista Lasallista de investigación. 2005; 2(1): 43-48.

5. Leonardo-redes. Cómo contribuyen los residuos al desarrollo sostenible y al cambio climático [internet]. [Consultado 3 Jul 2019]. Disponible en: https://www.leonardo-gr. com/es/desarrollo-sostenible-cambio-climatico-y-gestion-residuos

6. Conti R. Sintesi e caratterizzazione di carboni ottenuti dalla pirolisis di biomasse (biochar) per applicazioni in campo agricolo. [Yesi de laurea]. Boloña: Universidad di Bologna, Italia; 2013

7. Zhao L, Cao X, Masek O, Zimmerman A. Heterogeneity of biochar properties as a function of feedstock sources and production temperatures. J Hazard Mater. 2013; 256257: 1-9.

8. Roth C. Micro-gasificación: Cocinar con gas procedente de la biomasa sólida y seca Una introducción al concepto de las tecnologías para combustión de gas-madera y sus aplicaciones para cocinar. 1ra edición. Tegucigalpa: Universidad Agrícola Panamericana Zamorano (Honduras); 2011 
504 Enmer Trujillo A., Christian Eduardo Valencia A., María Cecilia Alegría A., Alejandrina Honorata Sotelo M...

9. Sobrero MC, Ronco A. Ensayo de toxicidad aguda con semillas de lechuga (Lactuca sativa L.) En Castillo G. editores. Ensayos toxicológicos y métodos de evaluación de calidad de aguas. Estandarización, intercalibración, resultados y aplicaciones. Otawa, Canadá: IDRC, IMTA; 2004 p. 55-67

10. Zucconi F, Pera A Forte M, De Bertoldi M. Evaluating toxicity of inmature compost. BioCycle. 1981; 22:54-57.

11. Cuevas M, Rosaldo J, López J. Evaluación de la toxicidad de los suelos mediante bioensayos con semillas. En: Cuevas Mc, Espinosa G, Ilizaliturri CA, Ania Mendoza A. Métodos ecotoxicológicos para la evaluación de suelos contaminados con hidrocarburos. Primera edición. México: Secretaria de Medio Ambiente y Recursos Naturales; 2012. p. 87-106.

12. Pastor Y, Martínez MM, Valdés R. Construcción de una planta de producción de biochar a partir de purines. [Internet]. Cartagena: Universidad Politécnica de Cartagena, España; 2013. [Consultado 05 Jun 2019]. Disponible en: https://www.upct.es/ orientap/agrolola/ biochar.pdf

13. Carhuancho F, Ramírez, J, Guerrero J. Gestión ambiental de residuos avícolas mediante digestión anaeróbica para la producción de fertilizantes orgánicos líquidos. Anales Científico. 2015; 76(1): 125-132.

14. De Andrade CA, Bibar MPS, Coscione AR, Pires Amm, Soares AG. Mineralização e efeitos de biocarvão de cama de frango sobre a capacidade de troca catiônica do solo. Pesqui Agropecu Bras. 2015; 50(5): 407- 416.

15. Carhuancho F. Aprovechamiento del estiércol de gallina para la elaboración de biol en biodigestores tipo batch como propuesta al manejo de residuos avícola. [Tesis pregrado]. Lima: Universidad Nacional Agraria La Molina; 2012.

16. Guo M, Shen Y, He Z. Chapter 8. Poultry Litter-Based Biochar: Preparation, characterization and utilization. En: He Z. Applied Research of Animal Manure: Challenges and Opportunities beyond the Adverse Environmental Concerns. New York: Nova Science Publishers; 2012. p.169-202

17. Williams CM. Poultry waste management in developing countries. [Internet]. Food and Agriculture Organization of the United Nations (FAO). Poultry Development Review. Agriculture and Consumer Protection Department, Animal Production and Health Division, 2011. [Consultado 05 Jun 2019]. Disponible en: http://www.fao.org/ag/ againfo/themes/documents/poultry/PDR_environment_main.pdf

18. Cantrell Kb, Hunt PG, Uchimiya M, Novak JM, Ro KS. Impact of pyrolysis temperature and manure source on physicochemical characteristics of biochar. Bioresource Technol. 2012; 107: 419-428

19. Bedussi F. Valutazione delle potenzialitá del biochar come componente dei substrati di coltivazione. [Tesis doctoral]. Milano: Universitá degli Studi di Milano, Italia; 2016.

20. Mohan D, Pittman CU, Steele PH. Pyrolysis of wood/Biomass for Bio-oil:A Critical Review. Energy Fuels. 2006; 20(3):848-889. 